\title{
Interacción con los grupos de interés (Mipymes) mediante el servicio de extensionismo técnológico
}

\author{
Interaction with the interest groups (Mipymes) \\ through the technical extensionism service
}

Alfonso Ramón Chung Pinzás ${ }^{1}$

Recibido 11 de junio de 2018, Aceptado 13 de septiembre de 2018

Received: June 11, $2018 \quad$ Accepted: September 13, 2018

\begin{abstract}
RESUMEN
El presente artículo muestra los resultados de la ejecución del proyecto "Servicios de extensionismo tecnológico para mejorar la calidad y productividad de las micro y pequeñas empresas (Mipymes) manufactureras en Lima y Callao", el mismo que fue ejecutado por la Facultad de Ingeniería Industrial de la UNMSM (FII) y financiado en forma por INNOVATE PERÚ y la FII.

Para ello se realizó la formulación y validación de un modelo de Extensionismo Tecnológico aplicado a las Mipymes, para tales efectos se intervino a 52 empresas en donde se lograron resultados exitosos en cuanto al logro del interés y la mejora de sus sistemas productivos.

Como resultados principales de la presente investigación, se tiene lo siguiente: contar con un modelo de Extensionismo tecnológico, así como con procedimientos estandarizados, conocer las necesidades de las Mipymes, establecer alianzas estratégicas con otras instituciones fuera del Perú y finalmente, iniciar las actividades de una oficina de extensionismo tecnológico en una universidad pública.

Estos resultados son relevantes ya que las Mipymes constituyen un actor muy importante en la economía del país, también son organizaciones reacias al cambio y muy celosas con su información. Finalmente, esta experiencia constituye un punto de inicio para que otras universidades puedan replicarla, adaptándola a su entorno.
\end{abstract}

Palabras clave: Extensionismo, transferencia tecnológica, innovación.

\begin{abstract}
This article shows the results of the execution of the project "Technological extension services to improve the quality and productivity of manufacturing of micro and small enterprises (MSMEs) in Lima and Callao", the same one that was executed by the Faculty of Industrial Engineering of the UNMSM (FII) and financed in the form of INNOVATE PERU and the FII.

The formulation and validation of a model of Technological Extensionism applied to MSMEs was carried out, for this purpose, 52 companies were intervened in which successful results were achieved in terms of achieving interest and improving their production systems.

As the main results of the present investigation, we have the following: a model of technological Extensionism, standardized procedures, to know the needs of MSMEs, to establish strategic alliances with other institutions outside of Peru and finally to initiate the activities of a technological extension office in a public university. These results are relevant since MSMEs are a very important player in the country's economy, they are also organizations that are reluctant to change and very jealous with their information. Finally, this experience constitutes a starting point for other universities to replicate it, adapting it to their environment.
\end{abstract}

Keywords: Extensionism, technology transfer, innovation.

\footnotetext{
1 Universidad Nacional Mayor de San Marcos. Escuela Profesional de Ingeniería Industrial. Lima, Perú. E-mail: achungp@unmsm.edu.pe

* Autor de correspondencia: achungp@unmsm.edu.pe
} 


\section{INTRODUCCIÓN}

Las MIPYMES forman parte del grupo de interés de la FII, además constituyen un factor muy importante para el país, ya que constituyen el $98 \%$ del desarrollo de la economía nacional, asimismo contribuye en $42 \%$ a la producción nacional y con el $88 \%$ al empleo privado [1].

Sin embargo, MIPYMES tienen procesos productivos y operativos poco eficientes que no les permite ser más competitivos, llevándolas a caer en la informalidad, a comprar insumos de baja calidad, a la excesiva rotación de personal y por ende sus productos y servicios no cuentan con la calidad necesaria para ingresar a mercados más rentables.

De hecho, las MIPYMES, en su amplia mayoría trabajan de manera informal, lo que no los hace visible ni a la academia (que intenta estudiar su naturaleza), ni al estado que busca mayores impuestos [2].

Además, para lograr que las MIPYMES sean eficientes, competitivas y generen puestos de trabajo, es necesario que cuentes con los siguientes factores [3]:

a. Capacidad emprendedora.

a. Tamaño y capitalización.

a. Tecnologías adecuadas.

a. Formación y habilidades de los trabajadores.

a. Acceso a los mercados.

a. Infraestructura disponible.

a. Servicios que faciliten los procesos productivos.

a. Seguridad jurídica sobre la propiedad.

Entre otros.

Considerando el escenario anteriormente descrito, el problema de investigación formulado para el presente proyecto fue el siguiente ¿En qué medida se pueden trasplantar a las MIPYMES algunas formas de hacer negocio de las grandes empresas? Sobre todo, aquellas orientadas a mejorar la calidad de los productos y servicios, mejorar la eficiencia de los procesos, aumentar la satisfacción del cliente y aumentar la rentabilidad.

El objetivo propuesto para el desarrollo del proyecto fue: Determinar en qué medida se pueden trasplantar a las MIPYMES algunas formas de hacer negocio de las grandes empresas.
Es decir, lo que se busca en el proyecto es desarrollar y validar un modelo que permita introducir en la MIPYMES metodologías de trabajo que utilizan las grandes empresas a fin de mejorar su desarrollo, estas metodologías se basan en métodos de calidad y productividad, como 5S, Lean Six Sigma etc. Y por otro, lado métodos enfocados en la innovación productiva como Design Thinking.

Todo esto da pie a que la FII cuente con una unidad de Servicios de Extensionismo, la misma que pueda funcionar en forma autosostenida, luego de finalizado el proyecto y por ende el financiamiento.

El proyecto permitió validar un modelo que favorece la transferencia tecnológica a las MIPYMES, Asimismo se logró mejorar los procesos productivos de las MIPYMES involucradas, generando no solo beneficios económicos, sino también una conciencia de mejora continua.

Los principales beneficiarios fueron las MIPYMES intervenidas, ya que pudieron acceder a herramientas que les permitió mejorar sus procesos, así como aumentar sus clientes.

\section{INNOVACIÓN Y DESIGN THINKING}

La innovación es el proceso que se realiza con un enfoque sistémico, que requiere de asociaciones y vínculos entre las distintas áreas del conocimiento, para la implementación y el funcionamiento de mejoras en las formas de hacer las cosas sobre la base de ideas creativas y transformadoras, y que busca siempre extrapolar dichas mejoras con éxito, al mercado, los productos, los procesos y los servicios para obtener un impacto económico, social o ambiental [4].

Como se puede apreciar en la definición anterior, la innovación no es solo I+D, sino que cierra su círculo, ya que hace llegar a los consumidores aquella nueva propuesta que surge de este análisis.

Y es muy importante este punto, ya que, si la idea no llega al consumidor final, como un producto listo para su uso, entonces no tiene sentido todo el esfuerzo invertido en $\mathrm{I}+\mathrm{D}$, ya que solo quedaría dentro de un laboratorio y a nivel de prototipo.

Una de las herramientas claves para poder desarrollar la innovación es Desing Thinking (DT), el cual 
se puede definir como una forma de solucionar problemas, disminuyendo los riesgos y aumentando las probabilidades de tener éxito, para ellos toma las necesidades humanas como punto de partida y luego observa, crea prototipos y los prueba, logrando soluciones deseables desde el punto de vista humano, técnicamente factibles y económicamente viables [5].

Es decir, como un método de conocimiento de las necesidades y deseos de las personas de una manera tecnológicamente factible, viable y estratégica.

En esta definición se puede apreciar el énfasis que se coloca en las personas, es decir, los problemas que requieren innovación, o se podría decir también, las oportunidades de innovación, nacen de los problemas que afrontan las personas, no de lo que el innovador piense que puedan necesitar. Es por eso necesario explorar dichas necesidades, pero utilizando un método validado y con rigor científico, tal y como lo es DT.

Las fases para implementar DT son las siguientes (ver Figura 1):

A continuación, se detallará brevemente cada una de estas fases [6]:

1. Empatiza: esta primera fase comienza con un análisis mixto, es decir cuantitativo y cualitativo, se trata de comprender las necesidades del cliente, así como su entorno, sus sentimientos etc. En otras palabras, es ponerse en sus zapatos.

2. Define: en esta segunda etapa se revisa los resultados de la primera, y crea un usuario típico a fin de determinar en forma más exacta las necesidades puntuales del cliente, para ello es necesario quedarse con los temas que dan valor, a fin de proponer problemas que puedan resolverse con innovación, como se puede apreciar, este análisis es ya cuantitativo.

3. Idea: en esta parte se generan diversas ideas que puedan dar solución al problema planteado, no hay límite para ello, es algo parecido a una lluvia de ideas, además hay que tener en cuenta que muchas veces las ideas más extrañas pueden ser las más innovadoras. En esta fase se debe dar mayor importancia a la creatividad.

4. Prototipa: en esta etapa se lleva a la realidad las ideas planteadas anteriormente, para ello se construyen prototipos de tal forma que pueda verse en la realidad las soluciones planteadas y también poder descartar a aquellas que no pueden llevarse a la práctica.

5. Testea: el testeo representa la prueba de todos los prototipos anteriormente elaborados con el usuario, a fin de lograr un intercambio de ideas que permita mejorar las soluciones propuestas, así como elegir a aquella que será aplicada por el usuario.

Como se puede observar, esta metodología está basada en el método científico, y también en parte en el ciclo PHVA, relacionado a la mejora continua.

\section{MEJORA DE LA CALIDAD EN LAS EMPRESAS}

Para entender mejor el tema de la mejora de la calidad, a continuación, se repasará tres definiciones de ella, formuladas por los denominados "padres de la calidad":

Para Crosby calidad es: Ajustarse a las especificaciones o conformidad de unos requisitos [7].

Para Deming, es el grado perceptible de uniformidad y fiabilidad a bajo costo y adecuado a las necesidades del cliente [8].

Para Jurán, calidad es adecuación al uso o adecuado para el uso [7], además el diseño del producto debe satisfacer las necesidades del cliente [9].

En las definiciones de Deming y Jurán el lector puede notar el hincapié que hacen en la satisfacción

Fuente: Elaboración propia.

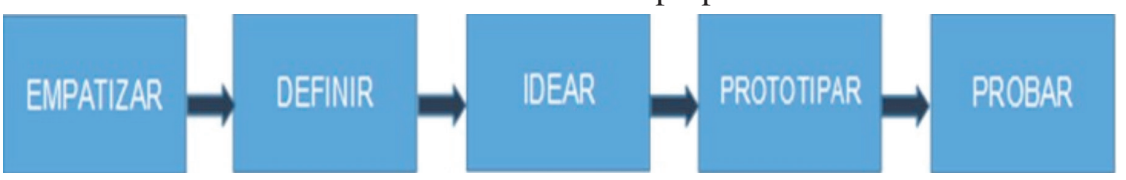

Figura 1. Fases de Desing Thinking. 
del cliente, de hecho, estas definiciones conciben la calidad en relación a las necesidades del cliente, aspecto que comparten con DT, mencionado en la sección anterior, por lo tanto, se puede apreciar en todo caso, que la medida de la calidad la pondría el cliente, en la medida de la satisfacción de sus necesidades.

Por otro lado, la definición de Crosby es quizás más general, ya que solo se refiere al ajuste o conformidad de unos requisitos, por lo tanto, estos requisitos pueden ser técnicos o relacionados a lo que busca el usuario.

Pero también hay que hacer notar que la definición de Deming, si bien está relacionada con la satisfacción del cliente, también contempla aspectos puntuales relacionados a la empresa u organización en general, sobre todo en lo relacionado a costos.

Esta definición, sin desmerecer las otras, se puede considerar como la que involucra los intereses de la organización con los del cliente, es decir, lograr la satisfacción de las necesidades del cliente, pero mediante procesos que representen un uso óptimo de los recursos de la organización.

En esta parte se está utilizando más el término de organización ya que las definiciones de calidad no solo son aplicables a empresas privadas, sino también a cualquier tipo de organización, incluyendo a aquellas que no tiene fines de lucro.

Para el presente artículo y para el proyecto que originó esta investigación e intervención en el grupo de interés (MIPYMES) de la FII, la definición de Deming es la que se consideró para su desarrollo e interpretación de resultados.

La mejora de la calidad en una organización se puede lograr mediante dos enfoques generales: la mejora continua y la reingeniería, a continuación, se va a definir brevemente cada uno de ellos.

Según Deming la administración de la calidad total requiere de un proceso constante, que será llamado Mejoramiento Continuo, donde la perfección nunca se logra, pero siempre se busca [10], hay dos puntos importantes que recalca el autor en esta definición, la primera es que la mejora continua (o mejoramiento continuo) es una actividad constante y perpetua en una organización que aplique gestión de calidad.

Esto significa que los procesos de dicha organización siempre deben ser analizados y mejorados, quizás en pequeñas mejoras, pero sin dejar este estilo de gestión.

Por otro lado, la definición de Deming, también introduce el término de excelencia cuando menciona que "la perfección nunca se logra, pero siempre se busca", en otras palabras, sumado a la actividad continua de buscar mejoras, también se debe tener presente que toda organización debe ir camino a la excelencia, aunque nunca la alcance, pero es en ese caminar en donde se va aplicando las mejoras a los procesos.

La mejora continua también se conoce como Kaizen [11], y se basa en el círculo PHVA, también conocido como la rueda de la calidad o el círculo de Deming, el mismo que se muestra en la Figura 2.

A continuación, y en forma resumida, se puede definir las etapas del PHVA como sigue [11]:

1. Planear: está relacionado con la formulación de los objetivos del sistema, así como sus procesos, esta etapa incluye la formulación del protocolo de implementación.

2. Hacer: implementar lo planificado con algún mecanismo de control.

3. Verificar: realizar el seguimiento y la medición de los resultados del sistema implementado y se compara con los esperados.

Fuente: Elaboración propia.

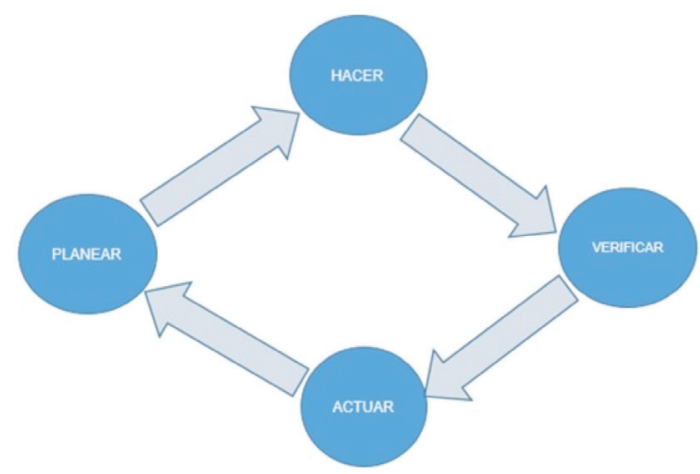

Figura 2. Círculo PHVA. 
4. Actuar: Si se ha logrado lo deseado, se documentan los cambios que hubo, en caso contrario se toma las acciones de mejora.

Una vez llegado a la fase cuatro, nuevamente se regresa a la fase uno, de tal forma que la rueda de la calidad es un proceso constante, y debe ser parte del quehacer de las organizaciones, es por eso la raíz de la mejora continua.

Por otra parte, se tiene a la reingeniería como otra forma de mejora, en este caso Hammer y Champy lo definen como la reconsideración fundamental y el rediseño radical de los procesos de negocios para lograr mejoras espectaculares en las mediciones críticas contemporáneas de desempeño, como costo, calidad, servicio y velocidad [11].

En esta definición se puede notar que la reingeniería representa un cambio radical de los procesos de la organización, es un comenzar de cero, un cambio revolucionario, es desechar de golpe todo lo antiguo y comenzar con algo nuevo. La reingeniería representa pues una nueva visión de las cosas, un rompimiento brusco y radical de un determinado paradigma de cómo hacer algo.

Cualquiera sea el caso, mejora continua o reingeniería, el obstáculo principal será la resistencia al cambio, que es natural en el ser humano, y se traduce en la tendencia de querer preservar el status quo, es decir la situación inicial.

Romper con el status quo implica el romper con el paradigma vigente de cómo hacer las cosas, de hecho, el ser humano es paradigmático, y eso se ha podido evidenciar en las empresas intervenidas, a pesar de ello, existen diversas técnicas que permiten salir de la denominada "zona de confort" y explorar nuevas alternativas de solución.

Por lo tanto, la implementación de cualquier sistema de mejora, no garantiza de por si su permanencia a través del tiempo, es por eso necesario implementar mecanismos que contrarresten esta natural tendencia a volver al punto cero, o situación pre test.

Una vez que los trabajadores asimilen y hagan suya dicha mejora, se habrá dado un gran paso en su permanencia en el tiempo. Sin embargo, no se debe prescindir de los mecanismos de control, además, hay que tener en cuenta también que cuando esto llegue, dicha mejora se convertirá en un paradigma para la organización, algo que todos acepten.

Por lo tanto, cuando se convierta en paradigma, es de esperarse que venga otro método rival que busque desplazar a este paradigma, de tal forma que se produzca nuevamente la lucha de contrarios, y se establezca una nueva forma de hacer las cosas, ya sea en forma radical (reingeniería) o progresiva (mejora continua).

En una organización de calidad este proceso de lucha entre paradigmas debería ser constante, porque con ello se segura la mejora de los procesos, siendo esto pues la principal constante de una filosofía de calidad.

En las empresas intervenidas a lo largo del desarrollo del proyecto se aplicó en todas, el esquema de la mejora continua, mediante $5 \mathrm{~S}$, radar de productividad entre otros.

\section{METODOLOGÍA DE DESARROLLO DEL PROYECTO}

La metodología se basó en las fases que se ilustran en la Figura 3.

La selección de los extensionistas se hizo en base a su experiencia en campo, así como a los conocimientos en calidad, productividad e innovación; este factor es importante ya que permitió luego ser reforzado con el entrenamiento propio del proyecto.

En entrenamiento en la formulación del modelo se basó primeramente en el reforzamiento de los conceptos fundamentales de calidad, productividad e innovación, así como en la introducción de los temas relacionados a Extensionismo tecnológico.

La formulación del modelo fue un proceso de co-creación entre los consultores seleccionados y el equipo técnico del proyecto (junto con los extensionistas).

A fin de poder luego validar el modelo en campo, es decir, iniciar las intervenciones a las MIPYMES, fue necesario primeramente realizar un diagnóstico de 180 empresas a fin de tener una visión detallada de la situación pre test, para ello se trabajó conjuntamente con Georgia Manufacturing Extension Partnership 
Fuente: Elaboración propia.

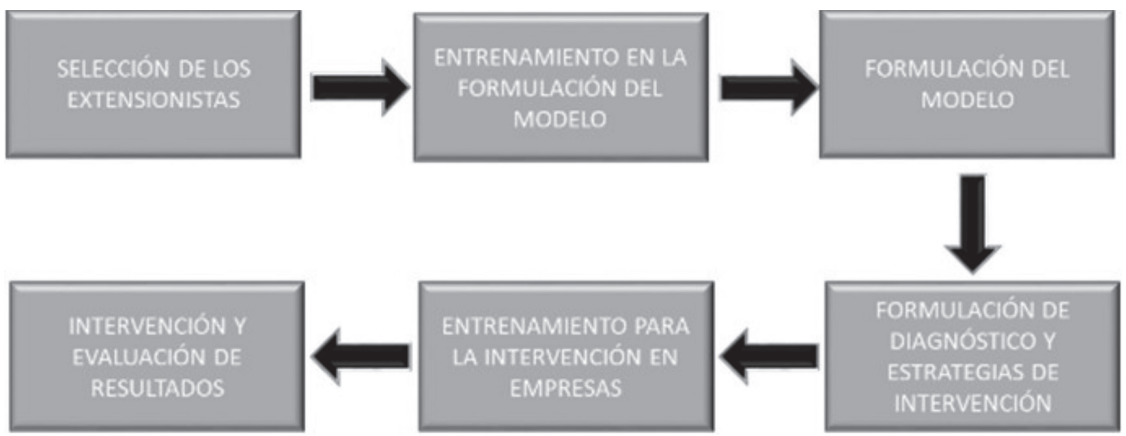

Figura 3. Fases del Proyecto.

(GAMEP), el cual es el programa de Extensionismo de Georgia Tech, lográndose con ellos una alianza estratégica, la misma que sigue vigente para otros proyectos.

Luego del diagnóstico, se trabajaron las estrategias para la intervención (también en conjunto con GAMEP) de tal forma que se tenga una guía para poder realizar los contactos con las MIPYMES.

El entrenamiento para la intervención de empresas, se realizó también mediante la co-creación, en este caso en colaboración con el Instituto Tecnológico de Paraná (TECPAR) cuyo servicio incluyó no solo la capacitación sino también el acompañamiento en la intervención.

En cuanto a la última fase, es decir la intervención, se logró intervenir a 55 MIPYMES, lo que representa una experiencia invalorable para la FII y para la implementación del servicio de Extensionismo en la UNMSM.

A continuación, se presenta en forma general, la metodología de intervención, cada una de las fases se documenta en un informe, según el formato respectivo, establecido dentro del proyecto:

1. En cada empresa MIPYME intervenida, se identificó el indicador problemático y la metodología de solución, para ello se hizo un muestreo preliminar, y con el empresario se preparó una propuesta de solución.

2. Al ser aprobada la propuesta, se coordinó con el empresario la hora de visita de cada sesión (procurando acordar la menor cantidad de sesiones), así como la persona encargada de liderar el proceso de mejora por parte de la empresa.

3. La siguiente etapa fue realizar un muestreo más exacto para obtener un promedio pre test del indicador problemático

4. Posteriormente se implementa la herramienta de mejora, según sea el caso

5. Luego, se muestrea nuevamente en el momento post test, de tal forma que se obtiene un valor del indicador problemático posterior a la implementación de la mejora

6. Finalmente se evalúan la diferencia de los promedios pre test y post test, a fin de determinar el grado de mejora, y se realiza el análisis inferencial respectivo.

7. Se cierra la intervención con el informe respectivo

Aparte de la intervención a empresas, también se logró la capacitación empresarios MIPYMES, mediante eventos presenciales, denominados Workshops y en forma virtual, mediante webinarios.

También se les hizo llegar material con información técnica tanto en forma presencial como virtual, de tal forma que se pudo reforzar las intervenciones y lograr nuevos clientes para el servicio de Extensionismo.

\section{RESULTADOS}

Los resultados esperados del proyecto fueron los siguientes:

1. Diagnosticar a 180 MIPYMES.

2. Al menos 40 MIPYMES que hayan tomado el servicio de Extensionismo. 
3. Capacitar a 180 MIPYMES en calidad, productividad e innovación.

4. Brindar información tecnológica a $\mathbf{1 8 0}$ MIPYMES.

5. Contar con un modelo de Extensionismo validado.

El periodo del proyecto fue de tres años, es decir durante los años 2015, 2016 y 2017, al momento de redacción del presente artículo se lograron los siguientes resultados:

Se diagnosticó a 180 empresas, obteniéndose información valiosa acerca de sus debilidades y necesidades.

Se intervino a 52 empresas, se logró capacitar y brindar información tecnológica a más de 180 empresas tanto en capación como en información tecnológica, superando la meta inicialmente propuesta.

Por otro lado, se cuenta con el modelo de Extensionismo, así como el manual operativo respectivo, lo que incluye los formatos respectivos para los informes, las técnicas de intervención a utilizar, el perfil de extensionista, entre otros.

\section{ANÁLISIS DE RESULTADOS}

Uno de los principales aspectos del proyecto es el análisis de los resultados de la intervención a las 52 empresas, en ese punto se pudo detectar que el problema que más se repetía era el de tiempo de producción, de hecho, representa el 37\% de los requerimientos recibidos (ver Tabla 1 ).

Como se puede apreciar en la Tabla 1, la diferencia entre los dos primeros lugares, es decir, tiempo de producción y unidades producidas, es de $25 \%$, además se alcanza un $70 \%$ acumulado con los cuatro primeros problemas del cuadro, esta información es importante ya que permite entender el patrón que sigue la muestra en cuanto a problemas.

Asimismo, la estrategia de solución que más ha demandado la fase de intervención ha sido la de $3 \mathrm{~S}$, que como se sabe es un preliminar a las $5 \mathrm{~s}$, es decir, clasificar, organizar y limpiar; solamente este primer paso logró mejorar los problemas que se presentaron en las organizaciones, además dejó sentada las bases para la implementación del resto de las 5S, la Tabla 2 muestra el detalle de las soluciones aplicadas.

En el cuadro anterior se puede apreciar que el mayor porcentaje de aplicación está dentro de las $3 \mathrm{~S}$, seguido de una diferencia bastante grande $(57 \%)$ de gestión y optimización de procesos, este panorama muestra que en las empresas intervenidas en su mayoría no tienen un sistema de producción ordenado, lo cual origina los problemas observados, de hecho un sistema desordenado trae como consecuencia, entre otros, excesivos tiempos de producción (lo cual se puede verificar en el cuadro 1 como problema principal).

Tabla 1. Problemas abordados.

\begin{tabular}{|l|c|c|c|}
\hline \multirow{2}{*}{ Cambios } & \multirow{2}{*}{ Cantidad } & \multicolumn{2}{c|}{ Porcentaje } \\
\cline { 3 - 4 } & & Individual & Acumulado \\
\hline Tiempo de producción & 19 & $37 \%$ & $37 \%$ \\
\hline Unidades producidas & 6 & $12 \%$ & $48 \%$ \\
\hline Inventarios & 6 & $12 \%$ & $60 \%$ \\
\hline Tiempos de entrega & 5 & $10 \%$ & $69 \%$ \\
\hline Nivel de ventas & 4 & $8 \%$ & $77 \%$ \\
\hline Merma & 3 & $6 \%$ & $83 \%$ \\
\hline Productos defectuosos & 3 & $6 \%$ & $88 \%$ \\
\hline Nivel de desperdicios & 3 & $6 \%$ & $94 \%$ \\
\hline Eficiencia del proceso productivo & 1 & $2 \%$ & $96 \%$ \\
\hline Tiempo de despacho en almacén & 1 & $2 \%$ & $98 \%$ \\
\hline Señalización & 1 & $2 \%$ & $100 \%$ \\
\hline Total & 52 & $100 \%$ & \\
\hline
\end{tabular}


Tabla 2. Servicios Brindados.

\begin{tabular}{|l|r|c|c|}
\hline \multirow{2}{*}{\multicolumn{1}{|c|}{ Servicio adoptado }} & \multirow{2}{*}{ Cantidad } & \multicolumn{2}{c|}{ Porcentaje } \\
\cline { 3 - 4 } & & Individual & Acumulado \\
\hline 3S & 36 & $69 \%$ & $69 \%$ \\
\hline Gestión y optimización de procesos & 6 & $12 \%$ & $81 \%$ \\
\hline Mejora de canales de comunicación & 3 & $6 \%$ & $87 \%$ \\
\hline Análisis de tiempos y movimientos & 2 & $4 \%$ & $90 \%$ \\
\hline 5s & 2 & $4 \%$ & $94 \%$ \\
\hline Gestión organizacional & 1 & $2 \%$ & $96 \%$ \\
\hline Lección de un punto & 1 & $2 \%$ & $98 \%$ \\
\hline Metodología de homologación & 1 & $2 \%$ & $100 \%$ \\
\hline Total & 52 & $100 \%$ & \\
\hline
\end{tabular}

\section{CONCLUSIONES Y RECOMENDACIONES}

Como conclusiones, se plantea las siguientes:

1. El problema que más se repitió en las empresas intervenidas ha sido el de tiempos de producción, lo cual se debe básicamente al desorden de los procesos productivos.

2. Es factible la implementación de un servicio de Extensionismo a MIPYMES en una universidad pública.

3. El impacto de la extensión tecnológica a una MIPYME es favorable.

4. El factor principal que aqueja a las MIPYMES es la falta de preparación y visión a futuro del dueño de la misma.

5. El extensionista debe tener un don de convencimiento hacia el empresario MIPYME a fin de que acepte el servicio, ya que al inicio son reacios.

Como recomendaciones se plantea las siguientes:

1. En el momento de la selección del extensionista, hay que evaluar también la disponibilidad y dedicación que le va a brindar al programa.

2. Un servicio de extensión tecnológica debe incluir un proceso de capacitación y sensibilización a fin de disminuir el nivel de rechazo del empresario MIPYME hacia estas técnicas.

3. Es necesario contar con un personal dedicado exclusivamente y a tiempo completo a la gestión administrativa del servicio de Extensionismo, sobre todo en una universidad pública.

4. Tratar de que el incentivo al personal sea lo más alto posible, dentro de lo permitido por la partida respectiva.

\section{AGRADECIMIENTOS}

Los logros del proyecto se deben al trabajo constante de un equipo técnico, el cual participó en forma comprometida con las metas planteadas y cuyos nombres se mencionan a continuación:

1. Dr. Jorge Inche Mitma.

2. Mg. María Huamán Mejía.

3. Lic. Calos Bermudes Mendoza.

4. Srta. Rosa Pingo Arrieta.

5. Srta. María Chumpitaz Palomino.

\section{REFERENCIAS}

[1] R. Regalado. "Las MIPYMES en Latinoamérica". Juan Carlos Martínez Coll. Primera ed. pp. 237. México D.F., México. ISBN. 2000. 8469065866.

[2] F. Portocarrero, B. Tarazona y L. Camacho. "Situación de la responsabilidad social empresarial en la micro, pequeña y mediana empresa en el Perú”. Universidad del Pacifico. Primera ed. pp. 9. Lima, Perú. 2006. ISBN 9972571033.

[3] C. Amat. "El Perú nuestro de cada día: nueve ensayos para discutir y decidir". Universidad del Pacifico. Primera ed. pp. 76. Lima, Perú. 2006. ISBN 9972570932.

[4] M. Ochoa, M. Valdés y Y. Quevedo. "Innovación, tecnología y gestión tecnológica". ACIMED. Vol. 6. No 4. 2007. ISSN 1024-9435.

[5] M. Serrano y B. Pilar. "Design thinking: Lidera el presente. Crea el futuro". ESIC Editorial. Primera ed. pp. 11. Madrid, España. 2015. ISBN 8415986548. 
[6] M. Castillo-Vergara, A. Alvarez-Marin y R. Cabana-Villca. "Design thinking: como guiar a estudiantes, emprendedores y empresarios en su aplicación". Ingeniería Industrial. Vol. 35. $\mathrm{N}^{\circ}$ 3, pp. 301-311. 2014. ISSN 1815-5936.

[7] J. Almedariz. "Gestión de la calidad y de la seguridad e higiene alimentarias". Paraninfo S.A. Primera ed. pp. 23 Madrid, España. 2013. ISBN 8497324390.

[8] J. Celma. "ABC del gestor deportivo". INDE. Primera ed. pp. 127. Barcelona, España. 2004. ISBN 8497290364.

[9] J. Juran y J. Medina. "Juran y el liderazgo para la calidad: manual para ejecutivos". Ediciones Díaz de Santos. Primera Ed. pp. 107. Madrid, España. 1990. ISBN 848718944X.
[10] A. Ducuara y A. Manrique. "La cultura de la calidad bajo las normas ISO en las empresas de Neiva: investigación". Universidad Surcolombiana. Primera Ed. pp. 43. Neiva, Colombia. 2005. ISBN 9588154774.

[11] F. Gryna, R. Chua y J. Defeo. "Método Juran”. McGraw-Hill. Quinta ed. pp. 59. 209. México D.F., México. 2007. ISBN 9780072966626.

[12] P. Pérez, y F. Múnera. "Reflexiones para implementar un sistema de gestión de calidad (ISO 9001: 2000) en cooperativas y empresas de economía solidaria". Universidad Cooperativa de Colombia. Primera ed. pp. 50. Bogotá, Colombia. 2007. ISBN 9588325293. 